\title{
Catalytic decomposition of methane on Ni- containing catalysts prepared by heterophase sol-gel method
}

\author{
Maxim Popov ${ }^{l, 2^{*}}$, Vladimir Maximov ${ }^{1}$, and Viktor Kogan ${ }^{1}$ \\ ${ }^{1}$ N.D. Zelinsky Institute of Organic Chemistry Russian Academy of Sciences, 119991, Moscow, \\ Russia \\ ${ }^{2}$ Novosibirsk State Technical University, 630073, Novosibirsk, Russia
}

\begin{abstract}
The most promising catalysts for the catalytic decomposition of methane are nickel-containing systems, since nickel has high activity, thermal stability, thermal conductivity, and relatively low cost. In this work, catalysts were prepared by the heterophase sol-gel method and investigated using catalytic equipment. The catalysts showed high stability and lifetime during the catalytic decomposition of methane at temperatures ranging from 535 to $675^{\circ} \mathrm{C}$.
\end{abstract}

\section{Introduction}

In June 2018, Japan adopted the fifth Strategic Energy Plan, which envisages building a "hydrogen" society. To 2021, hydrogen strategies have been accepted in following countries such as Germany, France, Australia, South Korea, the United Kingdom, the Netherlands, the United States and Russia. Traditional methods for producing hydrogen, for example, such as electrolysis of water or steam reforming of methane, have a number of significant disadvantages. Electrolysis of water is a very energy-intensive process, and it only can be successfully implemented in the countries with cheap electricity. As a result of steam reforming of methane, a large amount of $\mathrm{CO}_{2}$ is formed, which is difficult to utilize. Therefore, it is necessary to develop alternative methods for hydrogen producing, for example, catalytic decomposition of methane [1]

$$
\mathrm{CH}_{4} \rightarrow \mathrm{C}_{\text {(solid) }}+2 \mathrm{H}_{2} \quad \Delta \mathrm{H}^{\circ}=75,6 \mathrm{~kJ} / \mathrm{mol} \text {. }
$$

This method is free-from emissions, like carbon oxides $\left(\mathrm{CO}_{\mathrm{x}}\right.$-free $)$, and can be operate with low process temperatures and production of a valuable by-product, carbon nanofibers, which can be used in various applications $[2,3]$.

To carry out this process, a catalyst that can fit the requirements such as sufficiently developed specific surface area, high catalytic activity, long lifetime and low cost is needed.

Ni-containing catalysts have already demonstrated their efficiency in this process [4]. Most of the studies in this field firstly pay attention to the composition of catalysts, and did not pay attention to the texture characteristics [5, 6]. In this study, we obtained the experimental results regarding the influence of textural characteristics on the activity in

\footnotetext{
*Corresponding author:popovmaxvik@gmail.com
} 
catalytic decomposition of methane over nickel-containing catalysts at temperature range $535-675^{\circ} \mathrm{C}$.

\section{Experimental}

\subsection{Preparation of the catalyst}

The catalysts were prepared using the following reagents: $\mathrm{Ni}\left(\mathrm{NO}_{3}\right)_{2} \cdot 6 \mathrm{H}_{2} \mathrm{O}, \mathrm{Cu}\left(\mathrm{NO}_{3}\right)_{2} \cdot 3 \mathrm{H}_{2} \mathrm{O}$, $\mathrm{C}_{8} \mathrm{H}_{20} \mathrm{O}_{4} \mathrm{Si}$ (Ethylsilicate-40, TEOS), ethanol, $\mathrm{HCl}$ и $\mathrm{HNO}_{3}$.

The catalysts were prepared via heterophase sol-gel method [7]. A typical preparation procedure is described below. A pre-prepared solution containing ethanol, distilled water, and hydrochloric acid was added in TEOS $\left(40 \mathrm{wt} . \% \mathrm{SiO}_{2}\right)$ at room temperature and stirred strongly. The solution was kept at room temperature for $72 \mathrm{~h}$.

The required portions of the crystalline hydrates of the nickel salt were calculated referred to pure nickel. The nickel nitrate complex was dissolved in a $25 \%$ aqueous ammonia solution. This solution was added to distilled water at room temperature under vigorous stirring:

$$
\mathrm{Ni}\left(\mathrm{NO}_{3}\right)_{2}+2 \mathrm{NH}_{4} \mathrm{OH} \rightarrow 2 \mathrm{NH}_{4} \mathrm{NO}_{3}+\mathrm{Ni}(\mathrm{OH})_{2} \downarrow
$$

The mixture was kept for $10 \mathrm{~min}$, and then the flaky-like precipitate was filtered off and washed with deionised water to remove ammonium nitrate. To obtain highly dispersed nickel oxide, the resulting precipitate was dried in air at $110^{\circ} \mathrm{C}$ for $1 \mathrm{~h}$ and calcined in air at $250^{\circ} \mathrm{C}$ for $4 \mathrm{~h}$ :

$$
\mathrm{Ni}(\mathrm{OH})_{2} \rightarrow \mathrm{NiO}+\mathrm{H}_{2} \mathrm{O}
$$

Then the obtained highly dispersed $\mathrm{NiO}$ was mixed with the hydralizate. The mixture was adjusted to the consistency of a homogeneous paste and dried in air at $150^{\circ} \mathrm{C}$ for $1 \mathrm{~h}$. After that, the mixture was reduced with hydrogen at $255^{\circ} \mathrm{C}$ :

$$
\begin{gathered}
\mathrm{NiO}+\mathrm{H}_{2} \rightarrow \mathrm{Ni}+\mathrm{H}_{2} \mathrm{O}, \\
\mathrm{Si}\left(\mathrm{OC}_{2} \mathrm{H}_{5}\right)_{4}+\mathrm{H}_{2} \rightarrow \mathrm{SiO}_{2}+\mathrm{C}_{2} \mathrm{H}_{5} \mathrm{OH}
\end{gathered}
$$

Table 1 shows the composition of the synthesized catalysts.

Table 1. Composition of catalysts.

\begin{tabular}{|c|c|}
\hline Designation & Composition, wt.\% \\
\hline Cat. 1 & $10 \mathrm{Ni} / 90 \mathrm{SiO}_{2}$ \\
\hline Cat. 2 & $50 \mathrm{Ni} / 50 \mathrm{SiO}_{2}$ \\
\hline Cat. 3 & $90 \mathrm{Ni} / 10 \mathrm{SiO}_{2}$ \\
\hline
\end{tabular}

\subsection{Physico-chemical methods of catalyst research}

Catalysts were studied using the low-temperature nitrogen adsorption method using Quantachrome NOVA 1000e instrument. Prior to analysis, the samples were degassed in vacuum at $300^{\circ} \mathrm{C}$ for $6 \mathrm{~h}$ to remove physically adsorbed gases and water.

The analysis of texture characteristics was carried out at a temperature of $77 \mathrm{~K}$ and relative pressures $\mathrm{P} / \mathrm{Po}$ of the adsorptive gas (nitrogen) in the range from 0.005 to 0.995 to obtain complete adsorption and desorption isotherms. The specific surface area was calculated using the Brunauer-Emmett-Teller (BET) method. To obtain the size distribution of mesopores, the Barrett-Joyner-Halenda method was used.

$\mathrm{X}$-ray studies of the phase composition of the catalyst samples were carried out on a $D R O N-4-07$ diffractometer using $\mathrm{CuK} \alpha$ radiation (wavelength $\lambda=1.54 \AA$ ). The $2 \theta$ angle range was 20 to $70^{\circ}$. 
To study the internal structure of the catalysts, particularly, the surface morphology, the transmission electron microscope JEOL JEM-2200 FS was used. The microscope was equipped with a $200-\mathrm{kV}$ gun with the Schottky-type cathode and built into the column, fully integrated energy filter which also allows for elemental and chemical analysis of samples.

\subsection{Studies of catalytic activity}

The catalytic activity in the course of thermocatalytic decomposition of methane was studied in a catalytic unit with flow tube reactor with fixed bed (volume was $65 \mathrm{~mL}$ ) at atmospheric pressure. The process temperature was ranged from 535 to $675^{\circ} \mathrm{C}$.

A catalyst with weight $0.026 \mathrm{~g}$ was placed on a fixed bed inside the reactor. Methane with a 99.99 vol.\% purity was fed with $39 \mathrm{~mL} / \mathrm{min}$ flow rate. The gaseous products were analyzed using gas chromatography.

\section{Results and discussion}

The results of textural characteristic analysis of the catalysts are shown in Table 2 .

Table 2. Texture characteristics of the catalyst.

\begin{tabular}{|c|c|c|c|}
\hline Sample & $\begin{array}{c}\text { Specific surface } \\
\text { area, } \mathbf{~ m}^{2} \mathbf{g}\end{array}$ & $\begin{array}{c}\text { Specific pore } \\
\text { volume, } \mathbf{~ c m}^{\mathbf{3}} \mathbf{g}\end{array}$ & $\begin{array}{c}\text { Average pore } \\
\text { diameter, } \mathbf{~ n m ~}\end{array}$ \\
\hline Cat.1 & 11.9 & 0.033 & 11.2 \\
\hline Cat.2 & 93.7 & 0.368 & 15.7 \\
\hline Cat.3 & 44.3 & 0.199 & 18.0 \\
\hline
\end{tabular}

Since the pore size influences on the catalyst activity, so with the pore radius decreasing the rate of the process increases as long as diffusion resistance begins to have an effect on. While the decreasing in the surface utilization somewhat compensates the effect of its increase with a decrease in the pore radius.

The catalytic activity depends on the high specific surface area of the catalyst, i.e. due to the large number of pores and free access of methane to active sites on the inner surface of the pores throughout the entire volume of the catalyst.

From the data shown in Table 2, it can be seen that the catalyst with the highest specific surface area is Cat. 2 catalyst with an average pore diameter of $15.7 \mathrm{~nm}$, which is closest to the mesopore range. This catalyst has a larger total pore volume compared to Cat.1 and Cat.3 samples. With this one would expect high catalytic activity of the Cat.2 sample. Diffraction patterns of samples Cat.1, Cat.2 and Cat.3 are shown in Figure 3. 


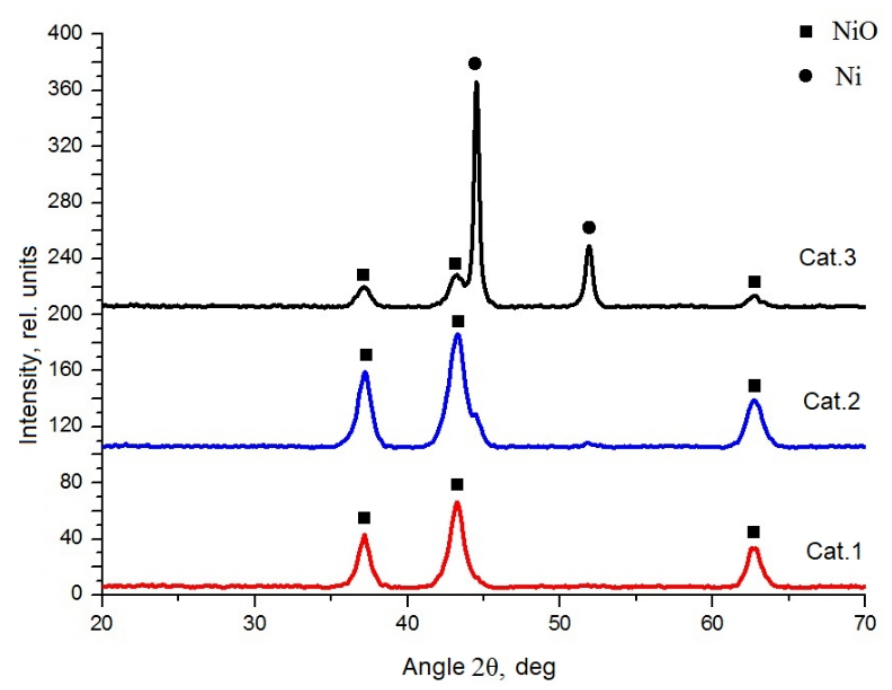

Fig. 1. Diffraction patterns of Ni-containing catalysts.

According to the X-ray diffraction data (Fig. 1), the presence of diffraction peaks corresponding to the crystalline phases of $\mathrm{Ni}$ and $\mathrm{NiO}$ was established in the composition of the catalysts. Silicon oxide was not shown because it is X-ray amorphous.

The average crystallite size was also determined (Table 3 ).

Table 3.Average crystallite size according to XRD data.

\begin{tabular}{|c|c|}
\hline Sample & Average crystallite size, $\mathbf{n m}$ \\
\hline Cat. 1 & 10.57 \\
\hline Cat.2 & 9.73 \\
\hline Cat.3 & 14.10 \\
\hline
\end{tabular}

According to XRD data, the samples Cat.1 and Cat.2 contain a rhombohedral phase of nickel oxide, while the sample Cat. 3 also contains a face-centered cubic phase of metallic nickel. TEM images of the sample with the highest surface area Cat.2 are shown in Fig. 2.
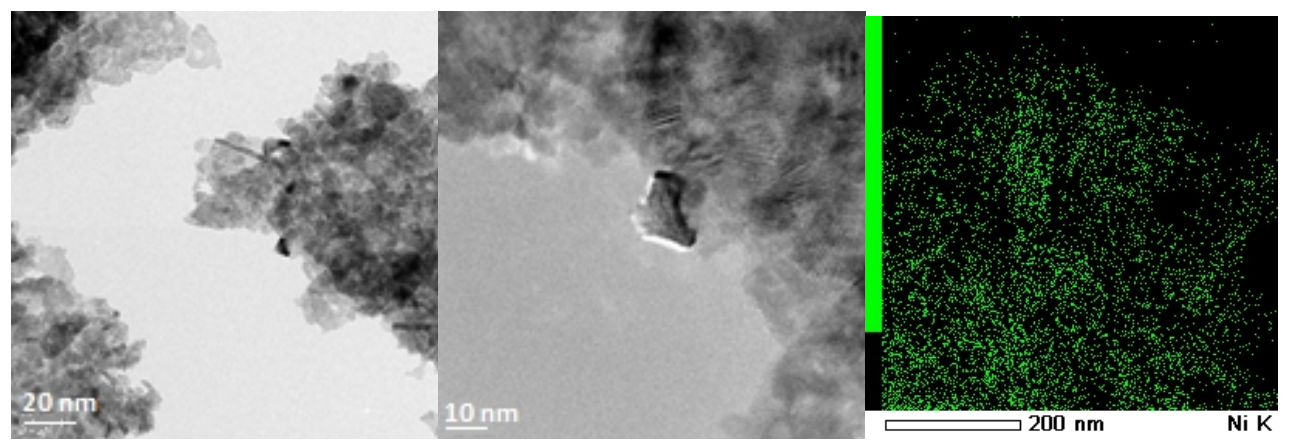

Fig. 2.Transmission electronmicrograph of catalyst Cat.2. 
As seen from the figure, the average size of nickel metal particles is $11 \mathrm{~nm}$, and the distribution of nickel particles on the support is uniform.

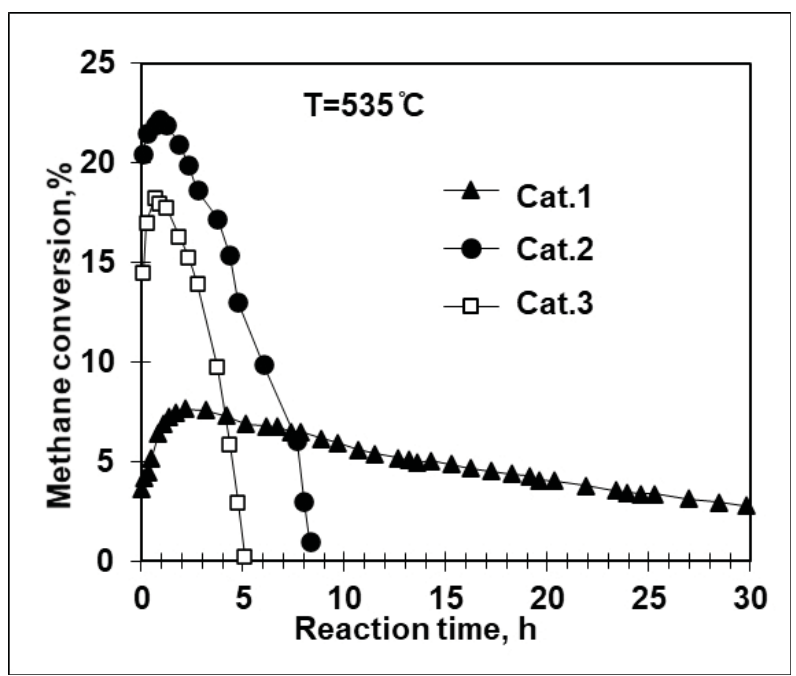

Fig. 3. Dependence of methane conversion on reaction time.

Studies of the catalytic activity of these samples (see Fig. 3) at $535^{\circ} \mathrm{C}$ have shown that the texture characteristics of the nickel catalyst have a greater effect on the conversion of methane than the content of nickel itself in the catalyst. Cat.2, which had the highest surface area and total pore volume compared to Cat.1 and Cat.3, had a maximum methane conversion at $23 \%$. However, Cat. 1 , with the smallest specific surface area and the smallest total pore volume, exhibited a long-life on flow with more than $30 \mathrm{~h}$ without the catalyst being deactivated yet.

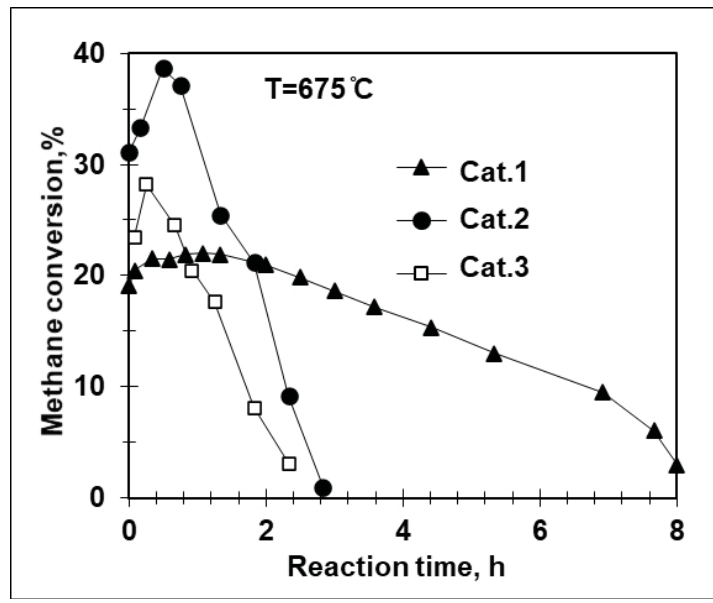

Fig. 4. Dependence of methane conversion on reaction time.

Increasing the reaction temperature to $675^{\circ} \mathrm{C}$ resulted in methane conversion increase , however, the lifetime of the catalysts significantly decreased compared to $535^{\circ} \mathrm{C}$ (see Fig. 4). Cat. 2 showed a maximum conversion at $38 \%$, while Cat. 1 showed a maximum catalyst life of about $8 \mathrm{~h}$. 


\section{Conclusions}

It has been shown that the heterophase sol-gel method can be used for synthesis of catalyst with an average particle size of $11 \mathrm{~nm}$, and it was found that the conversion of methane during methane thermocatalytic decomposition is more influenced by the texture characteristics of the catalyst than by the amount of nickel in the catalyst. It was found that the conversion of methane increases when increasing the temperature to $675^{\circ} \mathrm{C}$, independently on the textural characteristics and composition of the catalyst. While the maximum catalyst lifetime depends on specific surface area and much stronger, on the lower specific surface area and total pore volume of the catalyst.

Scientific Schools Development Program of Zelinsky Institute of Organic Chemistry is gratefully acknowledged.

\section{References}

1. M.A. Ermakova, D.Yu. Ermakov, A.L. Chuvilin, G.G. Kuvshinov, J. Catal. 201 (2001)

2. V.V. Shinkarev, A.M. Glushenkov, D.G. Kuvshinov, G.G. Kuvshinov, Appl. Catal. BEnviron. 85 (2009)

3. Y. L. Krutskii, M. V. Popov, A. A. Nikulina et al. Russ J Appl Chem. 90, iss. 9 (2017)

4. M.V. Popov, A.G. Bannov, Materials Today: Proceedings. 31 (2020)

5. Z. Fan, W. Weng, J. Zhou, D. Gu, W. Xiao, J. Energy Chem. 58 (2021)

6. Sh. Karimi, F. Bibak, F. Meshkani, A. Rastegarpanah, J. Deng, Y. Liu, H. Dai, Int. J.Hydrogen Energy. 46, iss. 39 (2021)

7. M. A. Ermakova, Ermakov D. Y. Catal. Today. 77, iss.3 (2002) 